\title{
POYNTING'S THEOREM AND THE DISTRIBUTION OF ELECTRIC FIELD INSIDE AND OUTSIDE OF \\ A CONDUCTOR CARRYING ELECTRIC CURRENT.
}

By William S. FrankLin.

INTRODUCTION.

PROFESSOR J. H. POYNTING has derived, ${ }^{1}$ from Maxwell's equations, an expression for the flow of energy in the electromagnetic field. According to Poynting the energy stream is, at each point, perpendicular both to the electric field and to the magnetic field, and the amount of energy which in one second streams across an area of one square centimeter at right angles to the stream is equal to $\frac{\mathrm{I}}{8 \pi} E H$, where $E$ is the electric field intensity and $H$ is the magnetic field intensity. J. J. Thomson ${ }^{2}$ has pointed out that, although Poynting's expression for the energy stream is perhaps the simplest expression that will satisfy the necessary conditions, it is not the only expression that will do so, inasmuch as any energy flow which has no convergence, or in which the energy streams are reëntrant, may be superposed upon the Poynting stream without changing the distribution of energy which actually takes place in any given case.

In his paper above referred to Poynting gives a number of examples illustrating his theorem. Thus he describes, in a general way, the flow of energy from the dielectric of a charged condenser to the various parts of a wire through which the condenser is discharged; he describes the flow of energy from the seat ${ }^{3}$ of the

${ }^{1}$ Phil. Trans., Vol. I75, Part II., p. 343, r884.

${ }^{2}$ Recent Researches, p. 31 3 .

${ }^{3}$ The author, for the purpose of his discussion, assumes this seat to be mainly at the surface of contact of acid and zinc. 
electromotive force in a voltaic cell to the various parts of the circuit to which the cell delivers current; and he describes the flow of energy from the hot junction of a thermoelement, showing how an excess of energy flows in at the cold junction thus producing the heating effect discovered by Peltier.

Poynting's derivation of his theorem is entirely satisfactory and convincing to one who is familiar with the use of differential equations in the discussion of electromagnetic theory, and the only uncertainty which would appear to affect the result is the uncertainty of the fundamental truth of Maxwell's equations.

The examples given by Poynting are not, however, entirely satisfactory, and for a sufficient reason. An example of the theorem is, in the nature of things, a solution of Maxwell's equations for a particular case. Such a solution requires a complete specification of all conditions at the boundary of the region in which the solution applies and a complete knowledge of all singularities occurring within this region. One who appreciates this fact is likely to be satisfied with the examples given by Poynting, notwithstanding their lack of numerical detail, for the solution of Maxwell's equations for any ordinary current generator and its circuit is impracticable.

In a series of short articles appearing at intervals from I897 to date, Mr. P. S. Wedell-Wedellsborg ${ }^{1}$ subjects Maxwell's equations in general and Poynting's theorem in particular to criticisms which have suggested to the writer the desirability of supplementing Poynting's original discussion in three particulars, namely, by stating as clearly as possible the conditions which determine the electric field distribution inside and outside of a conductor carrying an electric current, by working out the numerical details of several simple illustrative examples, and by calling attention to a mechanical model, due originally to Maxwell, which not only gives a good representation of an energy stream in an electro-magnetic field, but gives vivid

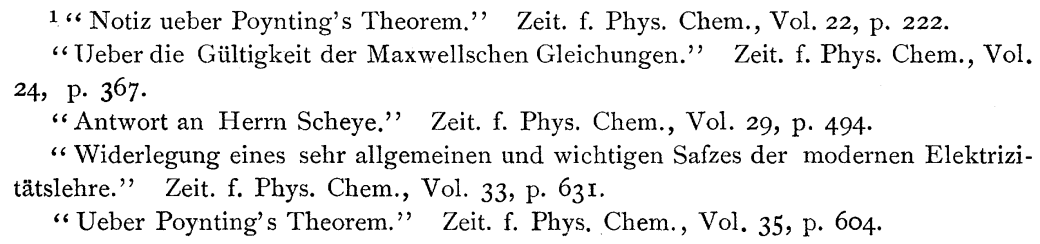


pictures of the steady electric current and of the action of an electric oscillator with the accompanying energy fluxes. The writer is of the opinion, however, that an understanding of Poynting's theorem and a conviction of its truth can be based only upon Maxwell's differential equations, and that particular solutions are not only generally impracticable, but useless as well. Still the discussion given in the following pages may be of interest to those who are pleased only when a mathematical discussion concludes with particular integrals and concrete results.

The earlier criticisms of Wedell-Wedellsborg are met, in a manner that seems to the writer satisfactory, by D. A. Goldhammer, ${ }^{1}$ and the need of a numerical solution of Maxwell's equations for a particular case involving the flow of energy into a conductor is well met by G. $\mathrm{Mie}^{2}$ who gives a solution for the case of two long parallel cylindrical conductors carrying equal and opposite currents.

Wedell-Wedellsborg's criticisms are quite largely based upon the question of the distribution of electric field inside and outside of the current conductor. In regard to this matter, A. Scheye ${ }^{3}$ points out that in most ordinary cases the effect of the energy of the electric field is very small and is ignored in the theory of current induction. In a later article Scheye ${ }^{4}$ meets two explicit objections raised by Wedell-Wedellsborg, namely, that the electric field distributions required by Maxwell's equations do not agree with experiment, and that, granting the existence of this electric field we are led to inconsistent results. Scheye agrees with the first objection in so far as to admit that further decisive experiments are required. The second objection he combats, pointing out that inasmuch as Maxwell's equations are consistent among themselves they cannot lead to inconsistent results if no foreign assumptions are introduced.

1 “"Einige Bemerkungen iiber die von Hernn Wedell.Wedellsborg vermŭtete Nichtgültigkeit der Maxwellschen Gleichungen für das Innere der Konduktoren."' Zeit. f. Phys. Chem., Vol. 23, p. 686.

2 " Ein Beispiel zum Poyntingschen Theorem." Zeit. f. Phys. Chem., Vol. 34, p. 522.

${ }^{3}$ Zeit. f. Phys. Chem., Vol. 26, p. 159.

${ }^{4}$ Zeit. f. Phys. Chem., Vol. 32, p. 145. 
On Electric Fieid Distribution.

Electric Field in the Conductor.-The electric field intensity at a point in a homogeneous conductor, of which the specific resistance is $r$, is equal to $r i$ when $i$ is the electric current density at the point. From this expression we can draw two important inferences, namely: (a) There is no free electric charge anywhere in the interior of a conductor carrying a steady electric current, for a steady electric current has no convergence, ${ }^{1}$ and therefore the field intensity $r i$ has no convergence if $r$ is constant. (b) Near the boundary of the conductor the electric field in the conductor is parallel to the surface of the conductor, for $i$ is parallel to the surface of the conductor, and so also is $r$.

Electric Field Outside of the Conductor.-The distribution of electric field outside of the conductor is determined by certain conditions which must be satisfied at the surface of the current conductor and at the surfaces of other conductors in the region. At each point of the surface of the current conductor the tangential component of the external electric field must be equal and parallel to $r i$ inside the surface, and at each point of the surfaces of other conductors the tangential component of the external field must be zero.

An immediate result of these conditions is that the electric lines of force are never normal to the surface of a current conductor, and that the electric lines of force may be parallel to the surface of the current conductor. In fact, however, it is only in exceptional cases that the electric lines of force are parallel to current conductors. Thus, in the particular case worked out by G. $\mathrm{Mie}^{2}$ the electric lines of force are very nearly perpendicular to the surfaces of the current conductors, and in one of the examples given below the lines of force are parallel to the surface of the current conductor. There is always at least one point on any electrical circuit where the electric lines of force are parallel to the current conductor, this is the point where the conductor has no surface charge.

1 That is, $\frac{d u}{d x}+\frac{d v}{d y}+\frac{d w}{d z}=0$, when $u, v$, and $w$ are the components of $i$.

${ }^{2}$ Reference given above. 
Reproduction by Stationary Charges of the Electric Field Distribution around a Current Conductor.-The distribution of electric field in the region surrounding an electric current, including the distribution inside of the conductor, can be reproduced by an arrangement of stationary electric charges, the electric current being reduced to zero. Imagine a given current conductor to have its specific resistance increased indefinitely, the size and shape of the conductor, and the generator electromotive force being unchanged. Then $r i$ remains unaltered while $i$ approaches zero. When the limit, $r=$ infinity, is reached the current is zero, the distribution of electric field and of electric charge is the same as at first, the electric charges are evidently stationary, and the magnetic field and energy stream have vanished. This ideal change of specific resistance of the current conductor might be realized by cutting the conductor into a very great number of lamina perpendicular to the lines of flow of the current, all the cross-cuts being made simultaneously.

That the electric charge which is distributed over a current conductor is stationary is further shown by the fact that these charges are surface distributions so that their movement would constitute current sheets in the surface of the conductor and there is no experimental evidence of the existence of such surface currents.

The Conception of Moving Lines of Force.-The idea of the side motion of the lines of force of the electric field surrounding a current conductor has been developed by Poynting, ${ }^{1}$ by J. J. Thomson, ${ }^{2}$ and by Wien, ${ }^{3}$ and this idea has been extensively used by other writers to give concreteness to discussions of electromagnetic action. The writer is of opinion that the fundamental notions of the partial derivatives with respect to time and space, such as form the basis of Maxwell's equations, are quite adequate to the full description of the phenomena of electromagnetic action; and that the only advantage of the idea of the side motion of the lines of

\footnotetext{
1 " The Connection between Electric Current and the Electric and Magnetic Induction in the Surrounding Field." Phil. Trans., Vol. 176, p. 277.

${ }^{2}$ Recent Researches, pp. I-50.

3 “Ueber den Begriff der Localizirung der Energie." Wied. Ann., Vol. 45, p. 685 .

"Ueber die Bewegungen der Kraftlinien im electromagnetischen Felde." Wied. Ann., Vol. 46, p. 352.
} 
force is its concreteness. In the case of a plane electromagnetic wave, for example, it seems to the writer to be quite as satisfactory to consider the rates of growth or decay of the electric and magnetic field intensities at a point in their relation to the space variations of these intensities in the neighborhood of the point, as to imagine an actual side motion of the lines of force although the latter idea is certainly better adapted to descriptive purposes. Either method appears to be applicable to any case inasmuch as Webster ${ }^{1}$ has recently derived Maxwell's equations from a consideration of the side motion of lines of force.

In applying the idea of the side motion of the lines of force to the case of a steady electric current a difficulty arises. The charges in which the electric lines of force terminate are stationary and it seems that the lines of force also must be stationary. When the electric lines of force are parallel to the surface of the current conductor their side motion involves no immediate inconsistency, but these lines of force may in every case be traced to termini in stationary charges at some more or less remote part of the current conductor.

In the case of unsteady electric currents, as for example, the transmission of electric waves along wires, ${ }^{2}$ the idea of side motion of the lines of force does not seem to lead to inconsistencies, for in this case the charges are not stationary.

Neglect of Electrical Energy in Ordinary Current Problems.-The energy associated with an electric current is in part the energy of the magnetic field and in part the energy of the electric field. In many cases the electrical energy is negligible in comparison with the magnetic energy. Thus, at a point distant one centimeter from a long copper wire one millimeter in diameter carrying a current of ten ampères, the magnetic energy is about 0.16 erg per cc. and the electrical energy is about $2 \times 10^{-12}$ ergs per cc. ${ }^{3}$ This example is somewhat misleading inasmuch as the magnetic energy falls off

\footnotetext{
${ }^{1}$ In a paper read before the American Physical Society, December, 1899.

${ }^{2}$ See paper by A. Sommerfeld, Wied. Ann., Vol. 67, p. 233. A number of diagrams are given in this paper from which a student can get a good idea of the trend of the electric and magnetic lines of force near a wire over which an electromagnetic wave is passing.

${ }^{3}$ This expression for the electrical energy is calculated from the component of the electric field parallel to the wire.
} 
rapidly as the distance from the wire increases while, in case of a long straight wire, the electrical energy does not fall off. This is equivalent to the statement that for short circuits the electrical energy is negligible while for extended circuits, such as long distance transmission lines, the total electrical energy may be as great as or even greater than the total magnetic energy.

Experimental Evidence of Electrical Field Surrounding an Electric Current.-The writer is not inclined to agree with Scheye ${ }^{1}$ that the experimental evidence as to the character of the electric field surrounding an electric current is to any considerable extent deficient. Ohm's law itself shows that the potential gradient parallel to a wire is equal to $r i$ and this law is verified by a great variety of experimental evidence. On the other hand, the so-called capacity effects on long transmission lines and in submarine cables are due immediately to the component of the electric field at right angles to the surface of the conductors; all practical calculations of these capacity effects are based directly or indirectly upon Maxwell's equations, and these calculations agree with experiment as closely as could be expected when we consider first that such calculations are usually abbreviated and that dielectrics and magnetic substances deviate from that beautiful simplicity of behavior which we usually assume as a basis of our calculations.

The submarine cable tests by Crehore and Squier ${ }^{2}$ constitute, perhaps, the most complete experimental verification, hitherto published, of the theory of the electric circuit involving both electric and magnetic energy.

\section{Examples Illustrating Poynting's Theorem.}

The following solutions of Maxwell's equations, illustrative of Poynting's Theorem, apply to two dimensions, so that the respective diagrams represent the solutions completely. The shapes of the conductors have been chosen with a view to simplicity and the electric lines of force and the energy stream lines belong to families of curves with which everyone is thoroughly familiar. A minimum of

1 Reference given above.

2 Presented in a paper read before the annual meeting of the American Institute of Electrical Engineers in May, 1900. 
effort of the imagination is therefore required for a full comprehension of these solutions and their value, as examples of Poynting's theorem is correspondingly great.

The Slow Discharge of Two Parallel Charged Cylindrical Conductors through a Cylindrical Current Sheet.-The only case, exemplifying Poynting's theorem, for which a complete solution of Maxwell's equations can be set up for all space, is the case of the discharge of a condenser. This problem has been treated in a variety of cases by Hertz ${ }^{1}$ and by J. J. Thomson. ${ }^{2}$ These authors have treated mainly the case of the oscillatory discharge. The case of very slow discharge is of course much simpler. This case is cescribed in a general way by Poynting ${ }^{3}$ as an example illustrating his theorem. The following is an exact solution of the problem of the slow discharge of two parallel charged cylinders through a cylindrical current sheet.

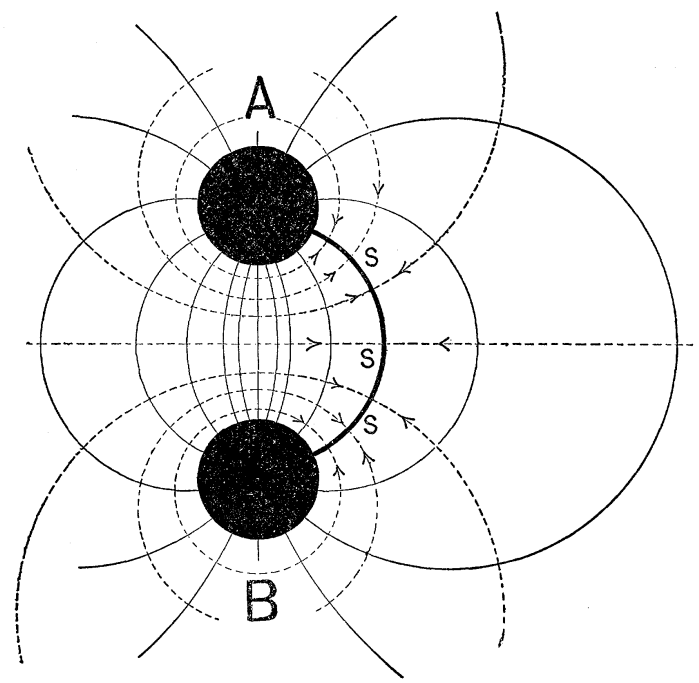

Fig. 1.

$A$ and $B$, Fig. I, represent two cylinders which are oppositely charged, and connected by a very high resistance sheet of metal SSS,

1 “Die Kräfte der electrischen Schwingungen, behandelt nach der Maxwell'schen Theorie." Wied. Ann., Vol. 36, p. r. A paper dealing with ar oscillating electrical doublet, the simplest case, perhaps, of a discharging condenser.

2 Recent Researches, pp. $25 \mathrm{r}-387$. The author treats of several types of electrical oscillators of finite dimensions.

3 Phil. Trans., Vol. I75, Part II., p $35^{\mathrm{I}}$. 
through which the cylinders are slowly discharged. The charges, which are uniformly distributed on the cylinders $A$ and $B$, produce an electric field of which the lines of force are circles, as represented by the full lines in the figure. If this electric field due to the charges on $A$ and $B$ happens to satisfy the necessary boundary conditions along the current sheet SSS, then this field alone will constitute the solution of the problem under consideration. If this field does not satisfy the boundary conditions along SSS, then charge will be distributed over the two faces of the sheet SSS in such a manner as to modify the electric field distribution and satisfy the boundary conditions along SSS. Since we are seeking the simplest possible solution we will assume that the resistivity $r$, of the sheet SSS per unit width (perpendicular to the plane of the paper) varies from point to point in such a way that $r i$ at each point of SSS is equal and parallel to the electric field at that point due to the charges on $A$ and $B$, where $i$ is the current per unit width of the sheet SSS. In this case the electric field due to the charges on $A$ and $B$ satisfies all necessary conditions, and the electric lines of force are circles touching $A$ and $B$ orthogonally. The magnetic field ${ }^{1}$ is everywhere perpendicular to the plane of the paper and the energy stream lines are circles cutting the electric lines of force orthogonally.

Current in a Cylindrical Sheet, Nearly Closed.-The above ideal example may be realized for the case of a finite electric current flow-

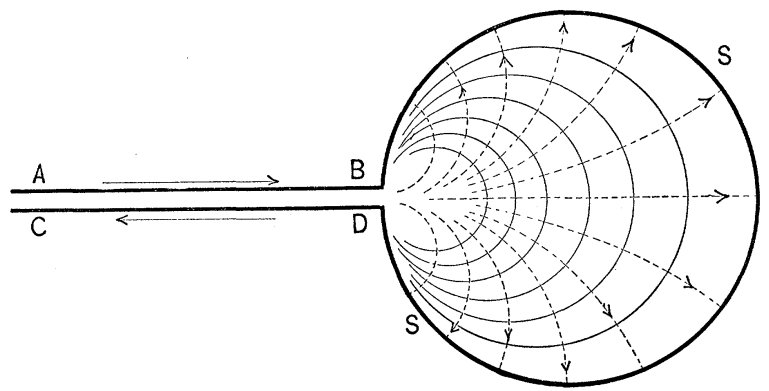

Fig. 2.

ing steadily in a cylindrical sheet as follows: Two plane current sheets $A B$ and $C D$, Fig. 2, are very near together and they separate

1 The magnetic field is not everywhere of the same intensity. In fact it grows less and less intense at increasing distances from the current sheet. The energy stream has diverg ence. 
at $B D$ forming a cylindrical shell $S S$. The electric current flows as indicated by the arrows and it is required to find the distribution of the electric field and of the energy stream inside of the shell. In this case the region in which the field distribution is to be determined is not a closed region, that is, it is not completely shut off by a conducting screen from the region occupied by the electric generator but since $A B$ and $C D$ are very near together the boundary conditions across the gap $B D$ may be represented by an electric doublet (line doublet). The problem is therefore determinate whatever the shape of $S S$ and whatever the manner of variation of the resistivity of the shell from point to point. The simplest case is that in which $S S$ is a circular cylinder. The lines of force from a linear doublet are circles and if $r i$ at each point of the shell is equal to the field intensity at that point due to the doublet at $B D$ then the boundary conditions are completely satisfied by the electric field due to the doublet alone and this, therefore, will be the actual electric field inside of $S S$. In this case there is of course no electric charge on the inner surface of SS. The electric lines of force are represented by the full line circles in Fig. 2 and the energy stream lines are represented by the dotted circles. These lines are not extended into

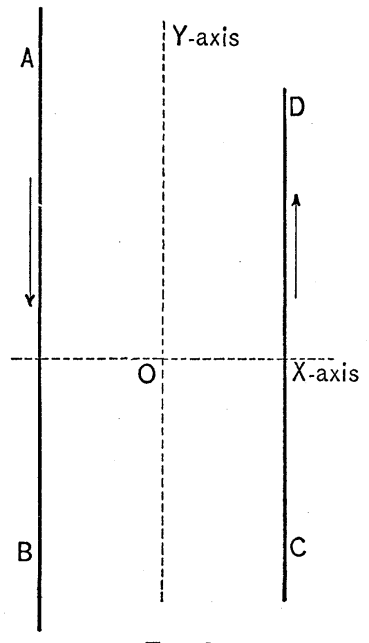

Fig. 3. the region immediately adjacent to the gap $B D$ inasmuch as the solution under consideration does not hold near $B D$. The magnetic field inside of $S S$ is uniform and the energy stream has no convergence.

Electric Current in Two Parallel Infinite Conducting Sheets.-Electric current flows down an infinite plane conducting sheet $A B$ and up an infinite conducting sheet $C D$ parallel to $A B$. The current per unit width in $A B$ and $C D$ is $i$, and the resistivity of each sheet per unit width is $r$. Required the distribution of the electric field and of the energy stream in the region between $A B$ and $C D$.

Choose the $Y$-axis midway between the planes and parallel to the electric currents. Choose the $X$-axis so 
that it cuts the two planes at points which are at the same potential. Let $X$ and $Y$ be the components of the electric field at any point. Then

$$
\frac{d X}{d x}+\frac{d Y}{d y}=0
$$

expresses the fact that the convergence of the electric field is zero throughout the region between $A B$ and $C D$.

The magnetic field is everywhere constant so that

$$
\frac{d Y}{d x}-\frac{d X}{d y}=0
$$

From equations (I) and (2) we have:

and

$$
\frac{d^{2} X}{d x^{2}}+\frac{d^{2} X}{d y^{2}}=0
$$

$$
\frac{d^{2} Y}{d x^{2}}+\frac{d^{2} Y}{d y^{2}}=0 .
$$

These equations permit separate solutions to be set up for $X$ and for $Y$.

In order that the boundary conditions for $X$ and $Y$ may be expressed as simply as possible let the distance between the planes $A B$ and $C D$ be taken as two units of length, so that the abscissas of the planes will be $-\mathrm{I}$ and $+\mathrm{I}$ respectively, and let the value of $r i$ be represented by the letter $a$.

The boundary conditions for $Y$ are :

$$
\begin{aligned}
& Y=-a \text { when } x=-\mathrm{I}, \\
& Y=+a \text { when } x=+\mathrm{I} .
\end{aligned}
$$

The solution of equation (4) under these conditions corresponds to the steady flow of heat ( $Y$ being temperature) through a homogeneous substance between two infinite planes $A B$ and $C D$, these planes being at temperatures $-a$ and $+a$ respectively. Therefore, $Y$, is a linear function of $x$ which has the prescribed values $\pm \mathrm{a}$ when $x= \pm \mathrm{I}$. Therefore :

$$
Y=a x .
$$

The boundary conditions for $X$ are determined with the help of 
equation (I) as follows: $Y$ being constant along $A B$ and along $C D$, $\frac{d Y}{d y}$ is zero along these planes. Therefore from equation (I) $\frac{d X}{d x}$ is zero along these planes. That is :

$$
\frac{d X}{d x}=\mathrm{o} \text { when } x= \pm \mathrm{I}
$$

The solution of equation (3) under these conditions corresponds to the steady flow of heat ( $X$ being temperature) through a homogeneous substance between two thermally insulating planes $A B$ and $C D$. Therefore $X$ is a linear function of $y$. That is :

$$
X=b y+c
$$

Equations (I) and (2) are not necessarily satisfied by solutions of (3) and (4). Equation (1) however has been used in finding the boundary conditions of $X$ from the given boundary conditions of $Y$, so that equation (I) is everywhere satisfied. ${ }^{1}$

The condition imposed by equation (2) fixes the value of $b$, equation (8). Thus, from (6) we have $\frac{d Y}{d x}=\alpha$ whence by equation (2) we have $\frac{d X}{d y}=a$; but by equation (8) $\frac{d X}{d y}=b . \quad$ Therefore $b=a$. The value of the constant $c$, equation (8), is determined as follows : The difference of potential between $A B$ and $C D$ at a distance $y$ above the $X$-axis is equal to $2 X$, or by equation (8) it is equal to $2 b y+2 c$, since the distance between $A B$ and $C D$ is 2 units. Therefore for $y=0$, this potential difference is $2 c$, but the $X$-axis has been chosen so as to pass through equipotential points on $A B$ and $C D$, so that $c=0$, and equation (8) becomes :

$$
X=a y .
$$

The differential equation of a line of force of the electric field is:

1 The two continuous functions $X$ and $Y$ satisfy equation (I) over the entire boundary to the region between $A B$ and $C D$. Therefore, according to the theory of functions, they must satisfy this equation throughout the region. The small number of disposable constants in the above solutions of equations (3) and (4) is due to the fact that the boundary conditions are fully consider ed in the thinking out of the solutions by the aid of the heat-flow analogy. 


$$
\frac{d y}{d x}=\frac{Y}{X}
$$

or, using equations (6) and (9),

of which the integral is

$$
\frac{d y}{d x}=\frac{x}{y}
$$

$$
x^{2}-y^{2}=C \text {. }
$$

The lines of force are, therefore, equilateral hyperbolas of which the asymptotes are the two straight lines $(y=x)$ and $(y=-x)$.

The magnetic field in the region between $A B$ and $C D$ is uniform and perpendicular to the plane of the paper in Fig. 3. The energy stream lines are, therefore, in planes parallel to the paper in Fig. 3 and since these stream lines are perpendicular to the electric field, the differential equation to a stream line is :

$$
\frac{d y}{d x}=-\frac{X}{Y}
$$

or, using equations (6) and (9),

$$
\frac{d y}{d x}=-\frac{y}{x}
$$

of which the integral is

$$
x y=C .
$$

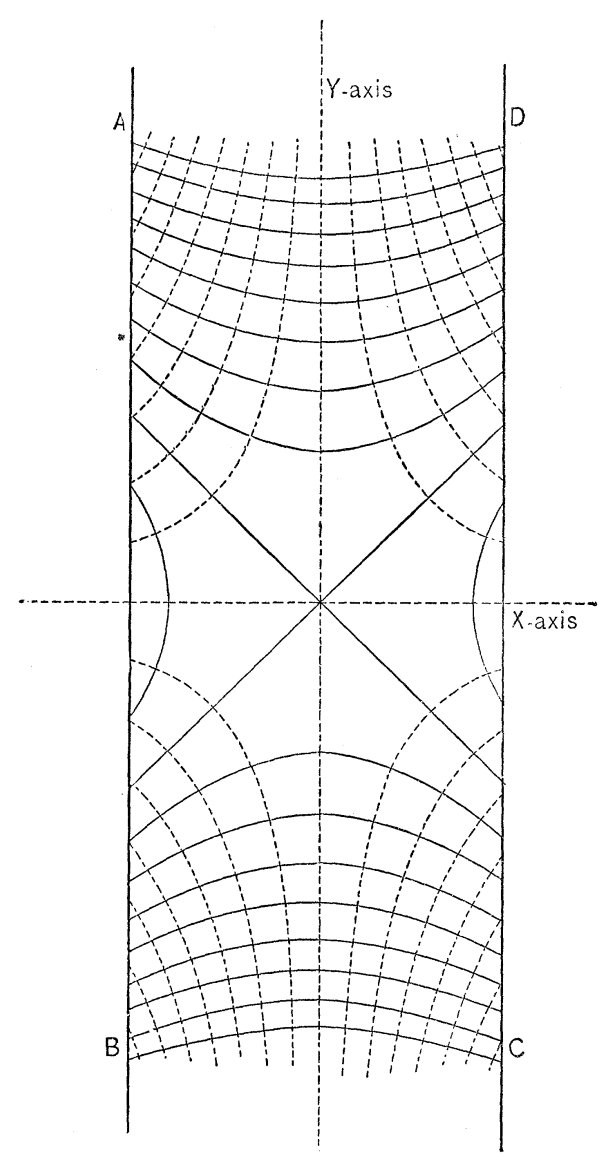

Fig. 4.

The energy stream lines are, therefore, equilateral hyperbolas of which the axes of reference are the asymptotes. 
Fig. 4 shows the electric lines of force and the energy stream lines. It is evident that the electric lines of force touch the conducting surfaces obliquely, except in the immediate neighborhood of the two points where the planes are cut by the $X$-axis. At these points the electric field near the planes is parallel to the planes, and the planes have no surface charge.

The above solution, as shown in Fig. 4, holds for a variety of conditions as follows :

I. Either conducting plane $A B$ or $C D$ may be replaced by another parallel plane at any distance $x^{\prime}$ from the $Y$-axis, provided the resistivity of the new plane is : $r=\frac{a x}{i}$; for, the value of $Y$ along the new plane, as given by equation (6), will be the value required by the boundary conditions, namely $Y=r i$.

2. A conducting plane, parallel to the $X$-axis and perpendicular to the paper in Fig. 3, may be connected across from $A B$ to $C D$ at any distance $y^{\prime}$ from the $X$-axis and the flow of current changed so as to come down on $A B$ to the new plane, thence across to $C D$, and up on $C D$, provided the resistivity of the new plane is $r=\frac{a y^{\prime}}{i}$; for the value of $X$ along the new plane, as given by equation (9), will be the value required by the boundary conditions, namely $X=r i$.

3. A conducting sheet of metal may be placed in the position ofany one of the equipotential surfaces (indicated by the energy stream lines in Fig. 4) without $g$ in any way disturbing the electric field, the magnetic field, or the energy stream. In particular a plane sheet of metal may be placed midway between $A B$ and $C D$ without producing any disturbance. This fact

Fig. 5. is made use of in a discussion, which follows, of the effect of a metal shield near a current conductor.

Flow of Energy Around a Metal Shield Placed near a Current Sheet.-An insulated metal plate $g h$, Fig. 5 , is placed as a shield near and parallel to a plane current sheet $A B$. The potential of the shield is equal to the potential at the point $d$ on $A B$, that is, the 
shield may be supposed to be connected to $A B$ by a wire $d d$. Required the distribution of the electric field and of the energy stream between the shield and the current sheet.

The required distribution is represented by the portion of Fig. 4 , which is between $A B$ and the $Y$-axis, the line $d d$, Fig. 5, being on the $X$-axis.

If the potential of the shield is equal to the potential of a remote point on $A B$, then the distribution of electric field and of energy stream between $A B$ and $g h$ will be represented by a portion of Fig. 4 between $A B$ and the $Y$-axis and entirely above or entirely below the $X$-axis. The distribution of electric field near the edges of $g h$ will be similar to the distribution of electric field near the edges of a small plate which is near a large plate, forming an ordinary air condenser. The character of the electric field in this case is, in a general way familiar to the reader, and the energy stream lines are of course perpendicular to the electric lines of force, so that the energy stream lines diverge into the surrounding open region near the edges of the plate $g h$, Fig. 5 .

When the potential of $g h$ is equal to the potential of $A B$ at a point between $g$. and $h$ the energy stream enters the shielded region at $g$ and at $h$, otherwise the energy streams into the shielded region at one end only, a portion of this energy flows into the conducting sheet $A B$, and the remainder streams out at the other end.

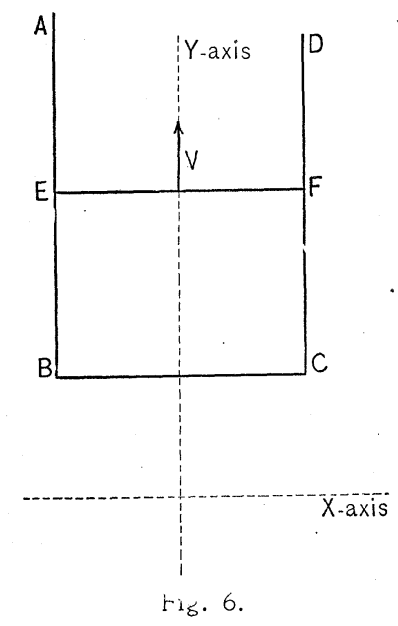

Flow of Energy in Case of an Induced Current.- $A B, B C$ and $C D$, Fig. 6, are conducting planes, $E F$ is a sliding plane in contact with $A B$ and $C D$, and the entire region is a uniform magnetic field perpendicular to the plane of the paper. The sliding plane $E F$ moves at low velocity $V$ which increases ${ }^{1}$ in proportion to its dis-

1 This velocity is assumed to be small so that the field distribution may everywhere accommodate itself to the changing conditions without unsteadiness. The velocity would have to be at each instant a little in excess of the value required simply to overcome the $\mathrm{i}$ ncreasing resistance of the circuit, on account of the fact that the inductance of the circuit $i$; increasing also. 
tance from $B C$ so that the electromotive force induced in $E F$ may suffice to maintain a constant current in the increasing circuit $E B C F$. It is required to find the distribution of the electric field and of the energy stream inside and outside of $A B C D$.

The curved lines in Fig. 7 represent the electric lines of force and the energy stream lines. The regions $A E F D, E B C F$, and the external region are treated separately.

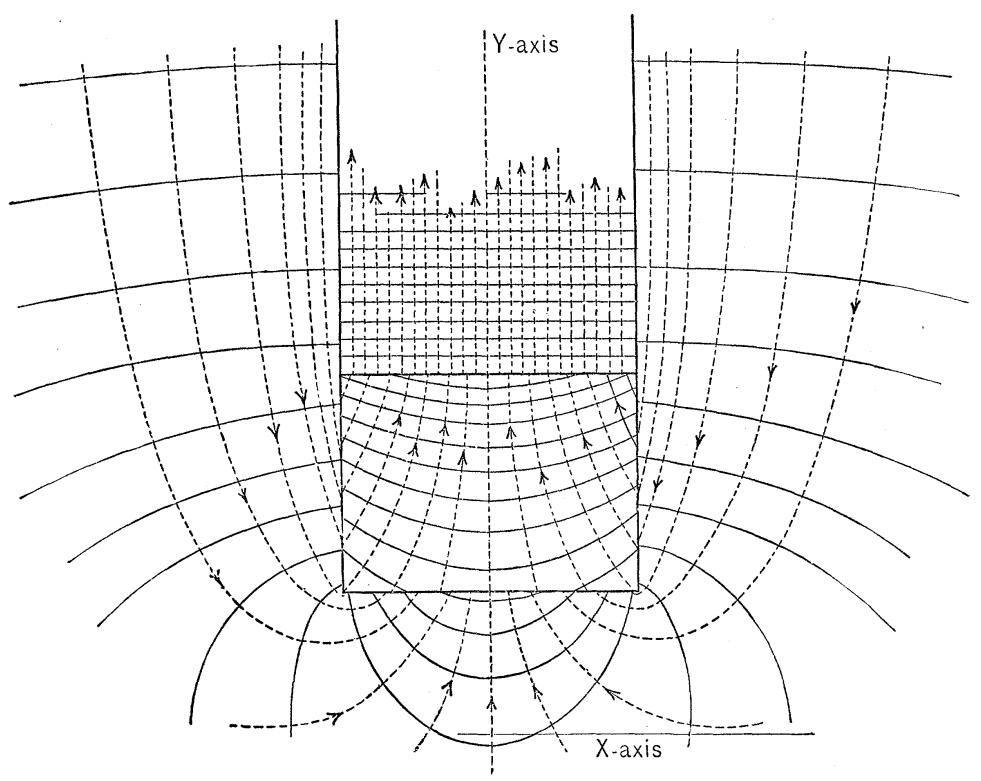

Fig. 7.

Let $e$ be the electromotive force induced in $E F$, let $r$ be the resistivity of the conducting planes per unit width, and let $i$ be the current per unit width of planes. Let $m$ be the length of $E F$, and let $l$ be the length $E B$ at the instant for which the solution is to be found. Let the axes of reference be chosen as shown in Fig. 6.

(a) In the region $A E F D$ the electric field is uniform and parallel to $E F$. The intensity of this field is $\left(\frac{e}{m}-r i\right)$; less than $\frac{e}{m}$ because of the loss of electromotive force in overcoming the resistance 
of the slide $E F$. The energy stream in the region $A E F D$ is uniform and parallel to $E A$.

(b) The distribution of the electric field and of the energy stream in the region outside of $A B C D$ is approximately as shown by the lines in Fig. 7. The energy stream passing up between $A$ and $D$ returns, as indicated by the dotted lines, outside of $A B$ and $D C$, and converges upon $E B, B C$ and $C F$. A portion of this energy stream changes into heat in the conductor and the remainder flows through the conductor into the region $E B C F$.

(c) The distribution of the electric field and of the energy stream in the region $E B C F$ is exactly the same as that shown by a portion of Fig. 4 as indicated by. the hyperbolic lines of force and lines of flow in Fig. 7, except that the energy stream is reversed in direction. The energy which streams up to $E F$ in the region $E B C F$ exceeds the energy which streams away from $E F$ in the region $A E F D$ by the amount $\left(m r i^{2}+2 l r i^{2}\right)$ which is the equivalent of the heat appearing in $E B, B C$ and $C E$, and is equal to $\left(P-m r i^{2}\right)$, where $P$ is the total power expended in moving the slides $E F$ and $m r i^{2}$ is the power equivalent of the heat appearing in $E F$.

The total fluxes of energy in the various energy streams above described may be calculated independently of a precise algebraic solution of the electric field distributions and the entire consistency of the above statements is easily shown.

The statements given above concerning the distribution of electric field and of energy stream in the two regions $A E F D$ and $E B C F$ are rigorously true as is easily seen from foregoing discussions given in this paper. On the other hand, the distribution in the region outside of $A B C D$ is only approximately represented in Fig. 7. The building up of algebraic functions for the electric field components $X$ and $Y$ in the external region so as to satisfy the boundary conditions over $A B C D$ is easily reduced to well known and soluble cases of heat flow, but the developments are more complicated than would be warranted in the present paper which is intended to be as simple as possible.

South Bethlehem, Pa., April, rgor. 